\title{
The Nature of the Giant Outbursts in the Bursting Pulsar GRO J1744-28
}

\author{
John K. Cannizzo ${ }^{1}$ \\ e-mail: cannizzo@lheavx.gsfc.nasa.gov \\ Goddard Space Flight Center \\ NASA/GSFC/Laboratory for High Energy Astrophysics, Code 662, Greenbelt, MD 20771 \\ ${ }^{1}$ Universities Space Research Association
}

Received __; accepted _ 


\begin{abstract}
We investigate the possible role of an accretion disk instability in producing the giant outbursts seen in GRO J1744-28. Specifically, we study the global, time dependent evolution of the Lightman-Eardley instability which can develop near the inner edge of an accretion disk when the radiation pressure becomes comparable to the gas pressure. Broadly speaking, our results are compatible with earlier works by Taam \& Lin and by Lasota \& Pelat. The uniqueness of GRO J1744-28 appears to be associated with the constraint that, in order for outbursts to occur, the rate of accretion at the inner edge must be within a narrow range just above the critical accretion rate at which radiation pressure is beginning to become significant.
\end{abstract}

Subject Headings: accretion disks: instabilities - X-rays: bursts - pulsars: individual (GRO J1744-28) 


\section{Background}

On 2 December 1995 the Burst and Transient Source Experiment (BATSE) on the Compton Gamma Ray Observatory (CGRO) discovered a new source which maintained a high persistent flux level, and also showed outbursts with a duration of about 10 seconds during which time the X-ray flux increased by about 4-5 (Kouveliotou et al. 1996). The source has subsequently been studied in detail by the Rossi X-ray Timing Explorer (=RXTE, cf. Swank et al. 1996). Subsequent investigation of this unusual source showed it to reside in a binary star system with an orbital period of 11.8 days (Finger et al. 1996). The pulsar has a spin period of 0.467 seconds (Finger et al. 1996) and concomitant corotation radius $1 \times 10^{8} \mathrm{~cm}\left(M_{1} / 1.4 M_{\odot}\right)^{1 / 3}$. Several papers have already appeared which attempt to address broad issues connected with the evolutionary status of the system and the strength of the magnetic field on the pulsar (e.g., Lewin et al. 1996, Daumerie et al. 1996, Lamb et al. 1996, Sturner \& Dermer 1996).

The question has naturally arisen as to the cause of the outbursts. The two main

models being discussed at present are (1) thermonuclear flashes of material accumulated on the surface of the neutron star, and (2) some as yet unspecified accretion disk instability which causes a periodic storage and dumping of some material in the inner disk. In the light curve one sees a dip and recovery period following each outburst, during which time the light asymptotically approaches its pre-outburst level. Also, the spectrum does not change dramatically going from quiescence to outburst. This behavior may not favor the thermonuclear flash model (Lewin et al. 1993, 1996).

The most obvious disk instability to invoke for the outbursts is the Lightman-Eardley (LE) instability (Lightman \& Eardley 1974). This instability develops in viscous accretion disks when the radiation pressure becomes comparable to the gas pressure. Several papers have already investigated the global, nonlinear evolution of accretion disks which are LE 
unstable (Taam \& Lin 1984=TL, Lasota \& Pelat 1991=LP). These investigators showed that the instability first develops at the inner edge of the disk and propagates outward as a heating wave of high viscosity material, much like the heating wave associated with the classical limit cycle instability in dwarf novae and X-ray novae. The instability does not propagate far, and the heated matter with its increased viscosity rapidly accretes onto the central star and produces a brief burst.

Figure 1 shows a $\sim 1$ hr stretch of data obtained 13 March 1996 with the Proportional Counter Array (PCA) instrument on RXTE. Three outbursts were seen - with durations of $\sim 10$ seconds and recurrence times of $\sim 1000$ seconds. The light curves shown in TL and LP bear a striking resemblance to those seen in GRO J1744-28, in particular one sees the broad dip and recovery phase after a burst has ended. The main shortcoming of the TL and LP bursts insofar as they might pertain to GRO J1744-28 is that they recur every few seconds and last for less than one second. These time scales are both much faster than observed. In their modeling TL and LP (1) set the viscosity parameter $\alpha$ to its maximal value of 1 , and they (2) took the inner disk edge to equal roughly the radius of the neutron star $\left(\sim 10^{6}\right.$ cm), whereas we suspect that in GRO J1744-28 the pulsar has a strong magnetic field. In this Letter we quantify the increases in the outburst time scales due to setting $\alpha$ to a more reasonable value, and to increasing $r_{\text {inner }}$.

\section{General Physical Considerations}

The limit cycle found by TL and LP is unlike the standard limit cycle for dwarf novae (see Cannizzo 1993a for a recent review). In the standard dwarf nova model, the locus of steady state solutions forms an S-curve when plotted as effective temperature versus surface density (Meyer \& Meyer-Hofmeister 1981). For the LE instability, however, 
there is no upper stable branch. (This situation has recently changed [Abramowicz et al. 1988].) Following the discovery of the LE instability in the 1970's, this lack of an upper stable branch was viewed as a severe limitation of the instability which might restrict its usefulness. The earliest time dependent studies of the LE instability solved only the viscous diffusion equation for surface density. TL and LP simultaneously solved both the diffusion equation and the thermal energy equation for temperature, and therefore considered rather more general accretion disks which need be neither steady (i.e., $\dot{M}$ constant with radius) nor in thermal equilibrium.

The fundamental obstacle to progress in accretion disk research has been the lack of understanding of the physical mechanism responsible for the viscous dissipation. In a recent study of the time dependent evolution of accretion disks in black hole X-ray binaries, Cannizzo et al. $(1995=\mathrm{CCL})$ found that, to reproduce the observed $\sim 30-40$ day exponential decays of the X-ray fluxes in the soft X-ray transients, the Shakura-Sunyaev viscosity parameter $\alpha$ must take the form $\alpha=\alpha_{0}(h / r)^{n}$, where $\alpha_{0} \simeq 50$ and $n=1.5$. In this study we adopt $\alpha=50(h / r)^{1.5}$, keeping in mind that this form may not be valid when radiation pressure begins to play a role. In the limit of large $\alpha$, we do not allow $\alpha$ to exceed 0.25 .

One may gain some rough understanding of the criterion for instability by considering scalings for physical properties at the local maximum in $\Sigma$ associated with the transition to radiation pressure domination. Using the Shakura-Sunyaev "middle" region for which $P=P_{\text {gas }}$ and $\kappa=\kappa_{\mathrm{es}}=0.34 \mathrm{~cm}^{2} \mathrm{~g}^{-1}$, the rate of accretion at which the gas and radiation pressure are equal is

$$
\dot{M}_{\text {crit }}=8.3 \times 10^{18} \mathrm{~g} \mathrm{~s}^{-1} \alpha^{-1 / 8} \Omega^{-7 / 8} \mu^{-1 / 2}
$$

(Shakura \& Sunyaev 1973). Here $\Omega$ is the local angular frequency (assumed to be Keplerian) and $\mu$ is the mean molecular weight $(=0.617)$. The temperature associated with the LE 
unstable branch and with $\Sigma_{\text {crit }}$ is $T_{\text {crit }}=2.11 \times 10^{6} \mathrm{~K} \alpha^{-1 / 4} \Omega^{1 / 4}$. Combining this with the law $\alpha=50(h / r)^{1.5}$ and the condition of hydrostatic equilibrium $h \Omega=\sqrt{\mathcal{R} T / \mu}$ gives

$$
\dot{M}_{\text {crit }}=1.5 \times 10^{18} \mathrm{~g} \mathrm{~s}^{-1} r_{8}^{1.26} m_{1}^{-0.55} \text {. }
$$

The viscous diffusion time at the inner edge $t_{\nu \text {, crit }}=r_{\text {inner }}{ }^{2} / \nu_{\text {crit }}=1200 \mathrm{~s} r_{8}^{0.58} m_{1}^{0.79}$, where $\nu_{\text {crit }}=\left(2 \alpha_{\text {crit }} / 3 \Omega_{\text {inner }}\right)\left(\mathcal{R} T_{\text {crit }} / \mu\right), r_{8}=r_{\text {inner }} / 10^{8} \mathrm{~cm}$, and $m_{1}=M_{1} / 1 M_{\odot}$. This time scale is roughly the observed recurrence time scale for outbursts in GRO J1744-28.

\section{3. $\quad$ Model}

The model we use is the same as that described in detail in TL and LP. This is a time dependent model which follows the evolution of surface density and temperature. The heating and cooling functions are evaluated separately, and the radial flow of energy - from both advection and diffusion - is included. The radial pressure gradient and departures from Keplerian flow are not included. Our numerical code is a modified version of the one used previously for modeling dwarf novae and X-ray novae (cf. Cannizzo 1993b, Cannizzo et al. 1995). In this study we utilize a grid spacing for which $\Delta r \propto \sqrt{r}$, and take $N=40$ radial grid points. For the pulsar mass we adopt $M_{1}=1.4 M_{\odot}$. The inner and outer disk radii are taken to be $r_{\text {inner }}=10^{7.5} \mathrm{~cm}$ and $r_{\text {outer }}=10^{9} \mathrm{~cm}$, respectively. The value of $r_{\text {inner }}$ was chosen to be less than the corotation radius, in order to be consistent with the observed spin-up of the pulsar. The value of $r_{\text {outer }}$ was chosen to be large enough so that the heating front never comes close to reaching it.

Figure 2 shows a sample light curve from our model, for a mass transfer rate into the

outer disk of $1.5 \times 10^{18} \mathrm{~g} \mathrm{~s}^{-1}$. We show a $3000 \mathrm{~s}$ history of (1) the rate of mass loss at the inner disk edge, and (2) the mass of the accretion disk. For this model, the observed burst 
durations of $\sim 10 \mathrm{~s}$ and recurrence times of $\sim 1000 \mathrm{~s}$ are reproduced. There is also a dip and recovery following each major outburst - although the dip is somewhat deeper and faster than observed.

Figure 3 shows the evolution of the disk in $Z=P_{\text {radiation }} / P_{\text {gas }}, \Sigma$, and $h / r$. We show 100 seconds of evolution centered on the second burst in Figure 2, spanning the time from 1480 to 1580 seconds. Each curve in Fig. 3 is separated by 2 seconds. The $t=0$ curve corresponds to just prior to the onset of the outburst. The ratio $Z$ has just begun to exceed $\sim 1$ in the inner disk. At slightly later times $Z$ increases rapidly to $\sim 10$ as the local gas heats and matter accretes onto the pulsar, but then quickly drops back down to $<1$ as the decreased surface density forces a return to the gas pressure dominated branch. The second panel in Figure 3 reveals how the heating front is propagated as a local enhancement in $\Sigma$. This is quite similar to what is seen in computations of the classical limit cycle instability (Cannizzo 1993b). Finally, the third panel shows that $h / r$ is always considerably less than unity - varying from a few percent in quiescence to $\sim 0.1$ in outburst.

Figure 4 shows the evolution of the disk at two radial grid points near the inner disk edge in $(T, \Sigma)$ space. The dashed line shows the standard equilibrium relation derived from taking the heating and cooling functions to be equal and assuming the viscosity to couple to $P_{\text {gas }}+P_{\text {radiation }}$ (as in our model). The dotted curve shows the equilibrium track for which the viscosity couples to the gas pressure only. In quiescence the evolutionary track closely follows the thermal equilibrium curve. The deviation between the actual evolution and the equilibrium curve becomes stronger for the evolution at $4 \times 10^{7} \mathrm{~cm}$ as $\Sigma_{\text {crit }}$ is approached. In Figure 4 we see that, after an annulus has made a transition to the LE state, it eventually migrates to smaller surface density (due to removal of matter from the inner edge), and finally proceeds back down to the gas pressure dominated branch. At this point we observe the build-up in $\Sigma$ associated with material from further out flowing in to refill the cavity. 
The evolution for the annulus at $1.1 \times 10^{8} \mathrm{~cm}$ is slightly different. This annulus is further away from the site of the initial trigger, and only makes the transition to the high state by virtue of having the heating front sweep past. The surface density drops after the heating wave passes, and rapid cooling forces a return to the gas pressure dominated branch. The evolutionary trends shown in Figure 4 are similar to those displayed by LP.

\section{Discussion and Conclusions}

We have shown how the LE instability operating in the inner edge of an accretion disk can be used to account for the giant outbursts seen in the bursting pulsar GRO J1744-28. In accordance with the time dependent calculations of TL and LP, we find that the LE instability never has a chance to go strongly into the non-linear regime because the rapid loss of material from the inner edge which happens once the inner disk starts to become LE unstable prevents $P_{\text {radiation }} / P_{\text {gas }}$ from running away to a high value. The material that is evacuated onto the pulsar during an accretion event is replenished by material flowing in from further out, hence the dip and recovery in the light curve following an outburst. TL and LP found much shorter outburst time scales (i.e., $t_{\text {recurrence }} \simeq$ a few seconds) than we do because they took larger $\alpha$ values $(\alpha=1)$ and smaller $r_{\text {inner values }}\left(\simeq 10^{6} \mathrm{~cm}\right)$.

The uniqueness of GRO J1744-28 might be associated with the possibility that $\dot{M}$ onto the pulsar could only exceed $\dot{M}_{\text {crit }}\left(r_{\text {inner }}\right)$ by some marginal amount - whose precise value remains to be determined - for the outbursts to occur. If the mass transfer rate were too large, there may exist a permanent region of radiation pressure domination at smaller radii, and not the oscillation we find between $P_{\text {radiation }}>P_{\text {gas }}$ and $P_{\text {radiation }}<P_{\text {gas }}$. For most low mass X-ray binaries it is probably the case that, lacking a strongly magnetized pulsar, the inner disk edge is close to the neutron star or black hole. The fact that persistent LMXBs 
such as Cyg X-2 and Sco X-1 are seen at all must mean that they have accretion rates comparable to GRO J1744-28. Therefore, since $\dot{M}_{\text {crit }}\left(r_{\text {inner }}\right)$ scales with $r_{\text {inner, one would }}$ expect $\dot{M} / \dot{M}_{\text {crit }}\left(r_{\text {inner }}\right)>>1$ for these systems, and outbursts of the type considered in this Letter could not occur. Further work must be done to determine how the size of the zone of instability varies with $r_{\text {inner }}$ and hence $\dot{M}_{\text {crit }}$.

One consequence of eqn. (2) is that, as the long-term, mean mass accretion rate onto the pulsar decreases and the persistent flux diminishes, the outbursts in GRO J1744-28 should cease. As of $\sim 1$ May 1996, plus or minus several days, with the persistent $R X T E$ PCA flux level at $\sim 0.1 \mathrm{Cb}$, the giant outbursts have indeed stopped occurring.

It is a pleasure to acknowledge useful criticisms from Charles Dermer. We also thank Jean-Pierre Lasota and Didier Pelat at the Meudon Observatory in Paris for useful discussions during a visit, and Laurence Taff, Alex Storrs, and Ben Zellner at the Space Telescope Science Institute for allowing us generous use of CPU time on their DEC AXP workstations. JKC was supported through the visiting scientist program under the Universities Space Research Association (USRA contract NAS5-32484) in the Laboratory for High Energy Astrophysics at Goddard Space Flight Center. 


\section{REFERENCES}

Abramowicz, M., Czerny, B., Lasota, J. P., \& Szuszkiewicz, E. 1988, ApJ, 332, 646

Cannizzo, J. K., 1993a, in Accretion Disks in Compact Stellar Systems, ed. J. C. Wheeler (Singapore: World Scientific), p. 6

Cannizzo, J. K. 1993b, ApJ, 419, 318

Cannizzo, J. K., Chen, W., \& Livio, M. 1995, ApJ, 454, 880

Daumerie, P., Kalogera, V., Lamb, F. K., \& Psaltis, D. 1996, Nature, in press

Finger, M. H., Wilson, R. B., \& van Paradijs, J. 1996, IAU Circular 6286

Kouveliotou, C., van Paradijs, J., Fishman, G. J., Briggs, M. S., Kommers, J., Harmon, B. A., Meegan, C. A., Lewin, W. H. G. 1996, Nature, 379, 799

Lamb, D. Q., Miller, C., \& Taam, R. E. 1996, preprint

Lasota, J.P., \& Pelat, D. 1991, A\&A, 249, 574

Lewin, W. H. G., Rutledge, R. E., Kommers, J. M., van Paradijs, J., \& Kouveliotou, C. 1996, ApJ, 462, L39

Lewin, W. H. G., van Paradijs, J., \& Taam, R. E. 1993, Space Sci. Rev., 62, 223

Lightman, A. P., \& Eardley, D. M. 1974, ApJ, 187, L1

Meyer, F., \& Meyer-Hofmeister, E. 1981, A\&A, 104, L10

Shakura, N. I., \& Sunyaev, R. A. 1973, A\&A, 24, 337

Sturner, S. J., \& Dermer, C. D. 1996, preprint

Swank, J., et al. 1996, IAU Circular 6291

Taam, R. E., \& Lin, D. N. C. 1984, ApJ, 287, 761 


\section{FIGURE CAPTIONS}

Figure 1. $\mathrm{A} \sim 1 \mathrm{hr}$ light curve in X-rays of GRO J1744-28 taken 13 March 1996 by the PCA instrument on RXTE. The persistent flux from the source was slightly less than 1

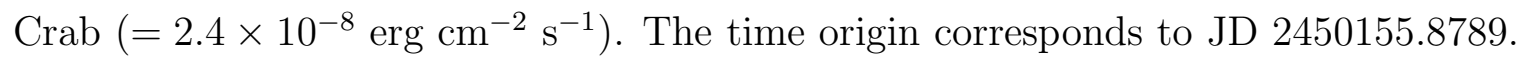

Figure 2. A sample 3000 second run from our model showing (a) the rate of mass loss from the inner disk onto the pulsar (which provides the accretion luminosity) and (b) the mass of the disk. The background accretion level into the outer disk is $1.5 \times 10^{18} \mathrm{~g} \mathrm{~s}^{-1}$.

Figure 3. The evolution of $Z=P_{\text {radiation }} / P_{\text {gas }}$, surface density $\Sigma$, and local aspect ratio $h / r$. The development shown spans the times $t=1480 \mathrm{~s}$ to $t=1580 \mathrm{~s}$ in Fig. 2. The dashed curves show the initial configuration of the disk, just before the outburst is triggered near the inner edge. The spacing between each curve is $2 \mathrm{~s}$.

Figure 4. The evolution of two annuli in $(T, \Sigma)$ space for one complete cycle. The two panels are for (a) $r=4.0 \times 10^{7} \mathrm{~cm}$, and (b) $r=1.1 \times 10^{8} \mathrm{~cm}$. The dashed and dotted curves give the equilibrium relations obtained by setting the heating and cooling functions

equal: $\Sigma^{2}=\left(32 a c T^{4}\right) /\left(27 \kappa \nu \Omega^{2}\right)$. The dashed curves are for $\nu$ scaling as $P_{g}+P_{r}$ as was used in the model, while the dotted curves are for $\nu$ scaling as $P_{g}$. 


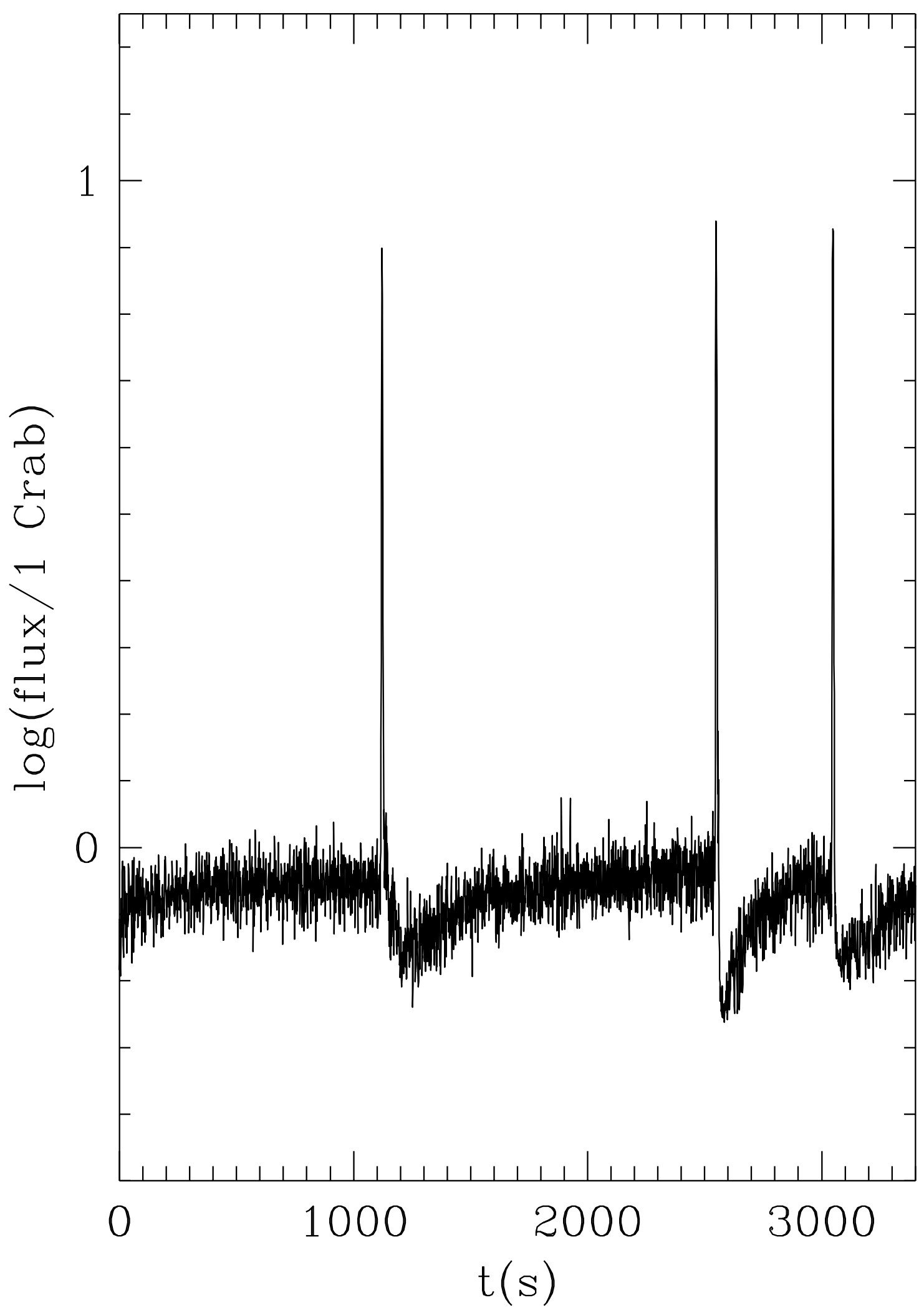




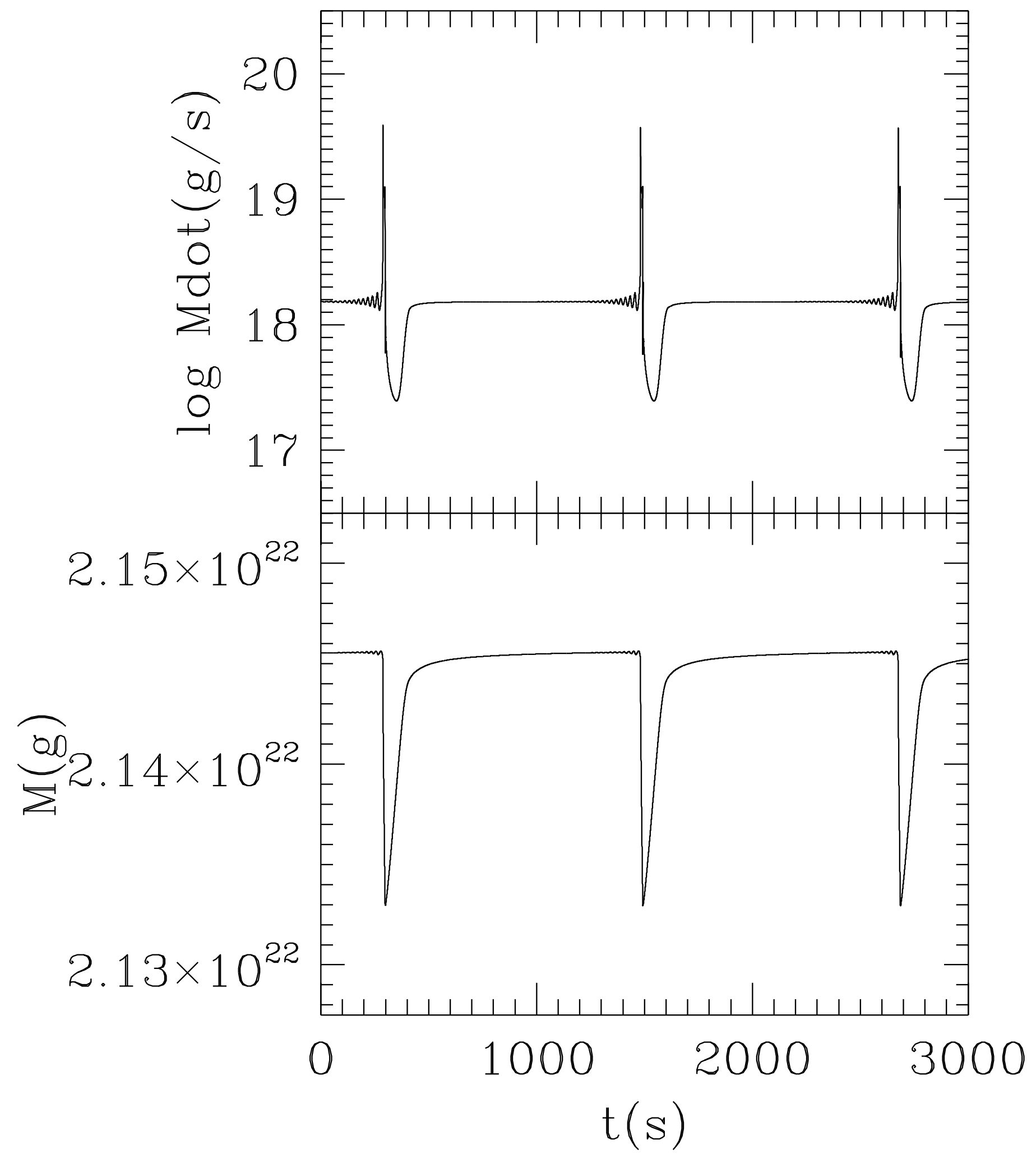




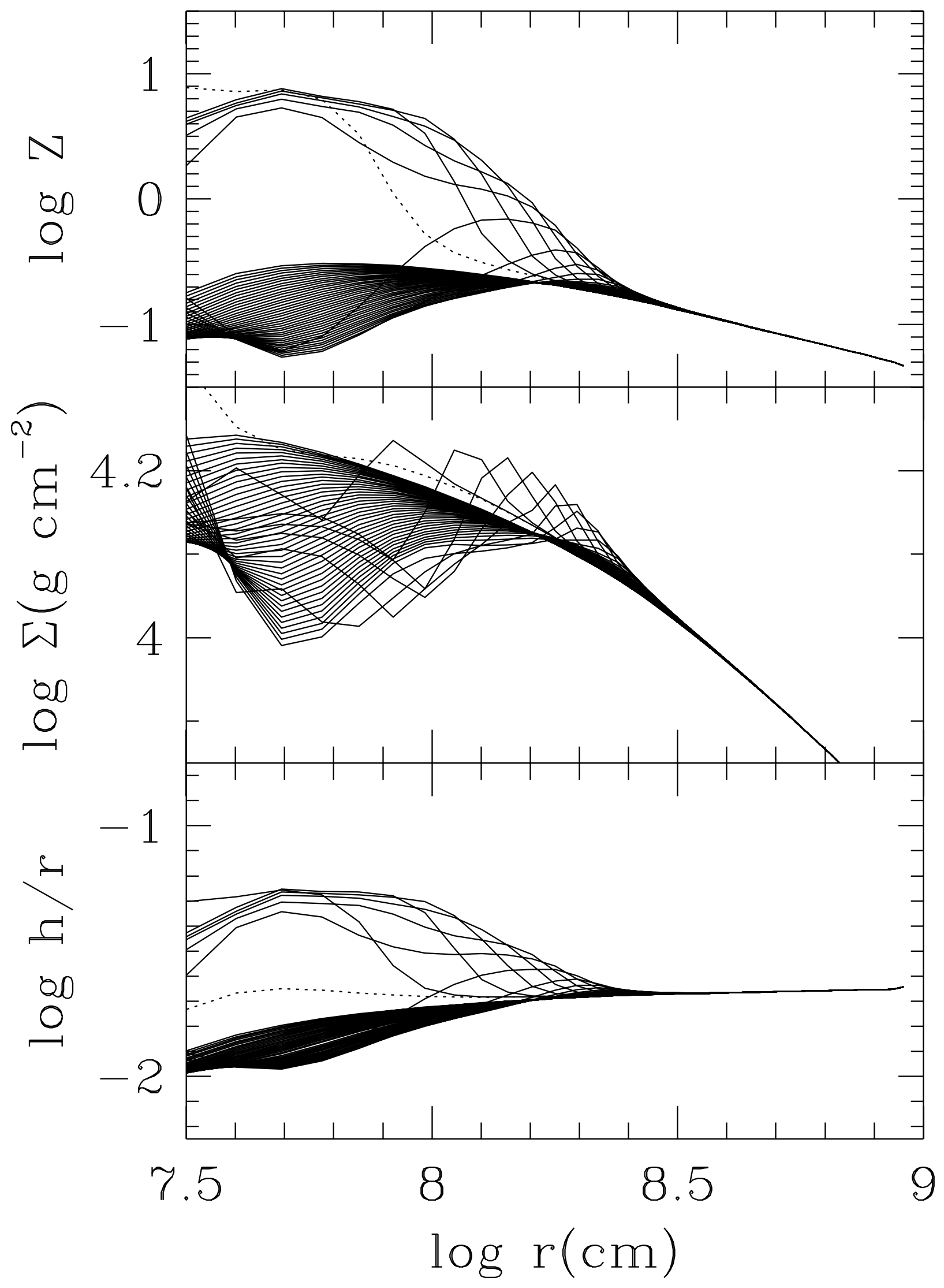




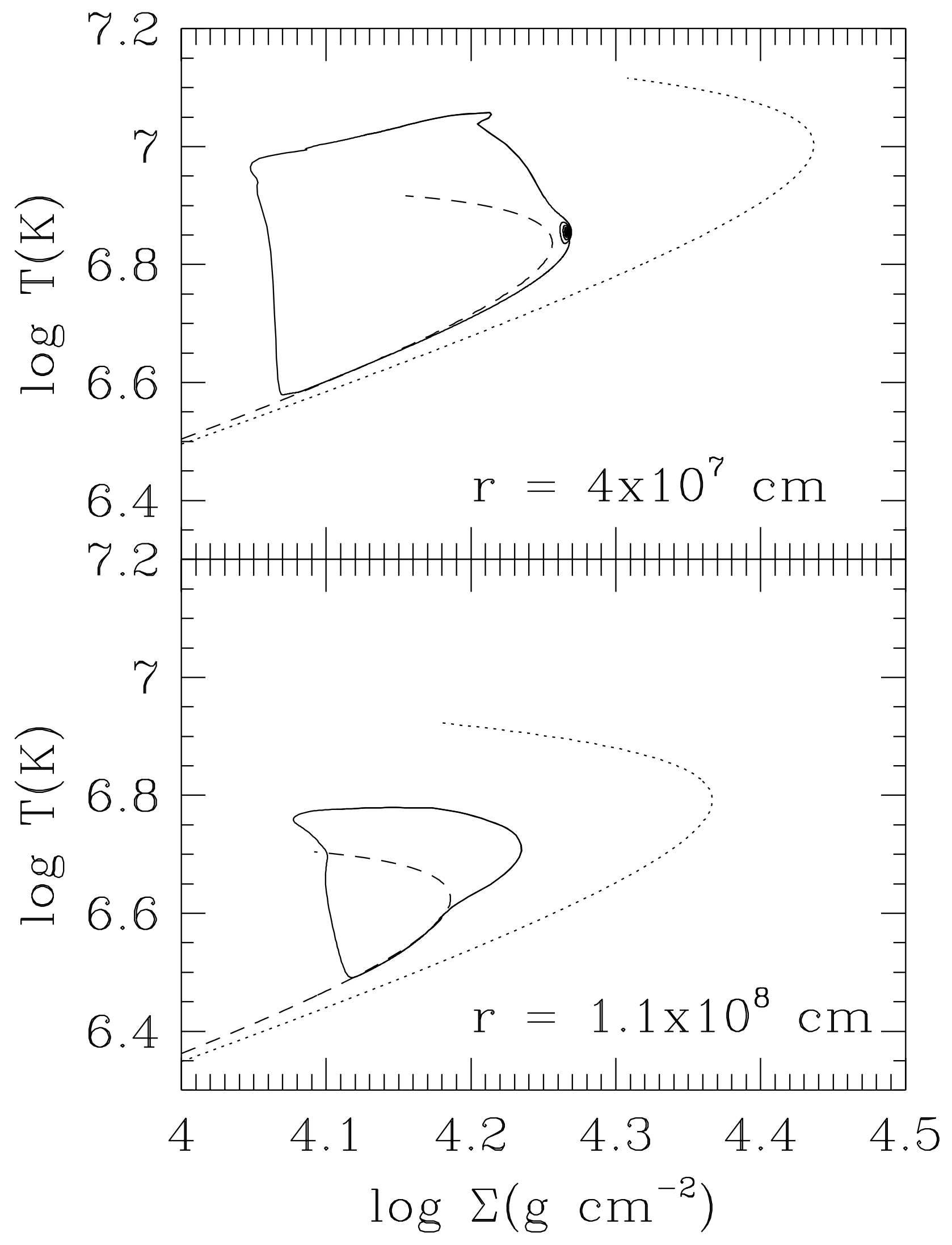

